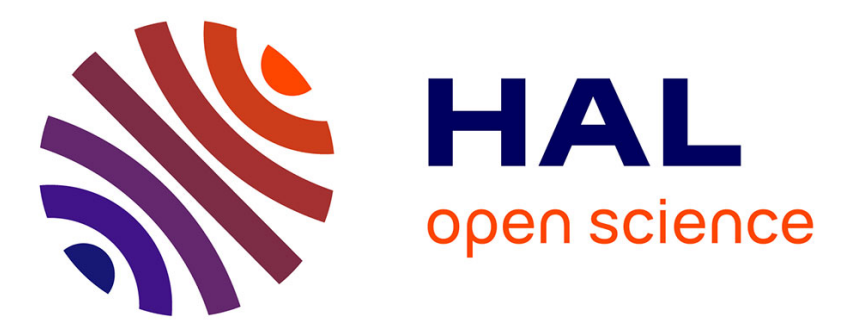

\title{
A Competency-Based Learning Resource Retrieval Process: The LUISA-UHP Case-Study
}

\author{
Monique Grandbastien
}

\section{To cite this version:}

Monique Grandbastien. A Competency-Based Learning Resource Retrieval Process: The LUISAUHP Case-Study. 9th TC3 World Conference on Computers in Education, IFIP TC3, Jul 2009, Bento Goncalves, Brazil. 10 p, 10.1007/978-3-642-03115-1 . inria-00423930v2

\section{HAL Id: inria-00423930 https://hal.inria.fr/inria-00423930v2}

Submitted on 13 Oct 2009

HAL is a multi-disciplinary open access archive for the deposit and dissemination of scientific research documents, whether they are published or not. The documents may come from teaching and research institutions in France or abroad, or from public or private research centers.
L'archive ouverte pluridisciplinaire HAL, est destinée au dépôt et à la diffusion de documents scientifiques de niveau recherche, publiés ou non, émanant des établissements d'enseignement et de recherche français ou étrangers, des laboratoires publics ou privés. 


\title{
A Competency-Based Learning Resource Retrieval Process : The LUISA-UHP Case-Study
}

\author{
Monique Grandbastien ${ }^{1}$ \\ ${ }^{1}$ LORIA, Université Henri Poincaré Nancy1, France \\ monique.grandbastien@loria.fr
}

\begin{abstract}
The paper describes a global framework enabling competency-based search of learning resources making a heavy use of semantic technologies. First it presents the generic components. Then it exemplifies how this framework can be adapted to a given environment, i.e. which knowledge representations, Web Services descriptions and other components have to be tailored or added to the framework. Then it shows step by step how a query is processed, Finally, users' feedback, lessons learnt and future trends are provided as well as comparisons with other approaches already published about annotating and retrieving learning objects in Learning Objects Repositories.
\end{abstract}

Keywords: Semantic based search, competencies, metadata, learning resource, ontology.

\section{Introduction}

The increasing number of digital learning resources available on the web has resulted in a growing interest for getting support for annotating, searching, retrieving, adapting and reusing such resources [14], [16]. Two main approaches are currently available today, namely the Web search (such as Google extended search) and the use of specialized "learning object repositories"(LOR) that provide search based on local metadata, such as Merlot [13]. In the first case there is a universal Web coverage, based on text, not specific to instruction or education. It uses links and not educational quality as the main selection criteria, and does not exploit information specific to learning/instruction. In the second case, the resource pool is limited, and some classification is provided, but it is local to the Web site. Some ongoing initiatives including [7], [4] aim at federating multiple sources but the query does not entail targeting the selection to the best ones. A common feature is that no domain or common sense knowledge is used and little support for queries on instructional properties is provided. Moreover the queries are mostly about topics and not about compentencies to be acquired.

Within this context, the LUISA project [12] (Learning content management system Using Innovative Semantic web Services Architecture) addresses the development of a reference semantic architecture (also called LUISA) for bringing solutions to the major challenges in the search, interchange and delivery of learning objects in a 
service-oriented context. This entails the technical description of the solution in terms of current Semantic Web Services (SWS) technology, and also the provision of the ontologies, facilities and components required to extend and enhance existing learning technology systems with the advanced capabilities provided by computational semantics.

The structure of the paper is as follows: in the next section we describe the global infrastructure provided by the LUISA project as well as its knowledge components. In section 3 we describe the adaptation of the LUISA framework to a university context for allowing competency-based learning resource requests. In section 4 we provide a working example illustrated by several screencopies from the application. Section 5 is devoted to the analysis of feedback from the first end-users and to possible improvements. Finally section 6 compares this work with other close initiatives and discusses possible roadmaps for the future in the field.

\section{A Semantic-Based Framework for Learning Resources Management}

The common framework is designed as a kernel from which applications tailored to fulfill the needs of a given institution are derived. It includes a general architecture based on semantic web services, and two ontologies, one based on [9] for describing learning resources, the other based on Human Resources competencies for describing the existing and targeted competencies of learners and resources. It also provides an annotation tool named eLUISA which has to be filled with data specific to the target application.

\subsection{Global Architecture}

This section describes the particular solution of the LUISA architecture (components, data structures and services) adopted for the realization of the current prototype. Figure 1 depicts the layered general architecture. The two middle layers represent the SWS-based infrastructure for e-Learning. The Process Language layer, service Abstraction layer and Data layer represent the actual interfaces of the SWSbased infrastructure. The Negotiation Layer provides the relationship between the end-users of the LUISA framework and the Semantic Web Service environment. There is a common functionality offered by the Negotiation component of the Negociation layer for the LUISA framework and the basic core implementation remains independent from case study particularities. The particularities of the case study are then not in the component itself, but in the Web service implementation that gives access to it and in the specific Query Resolver (another component in the Negociation Layer) developed for the case study. 


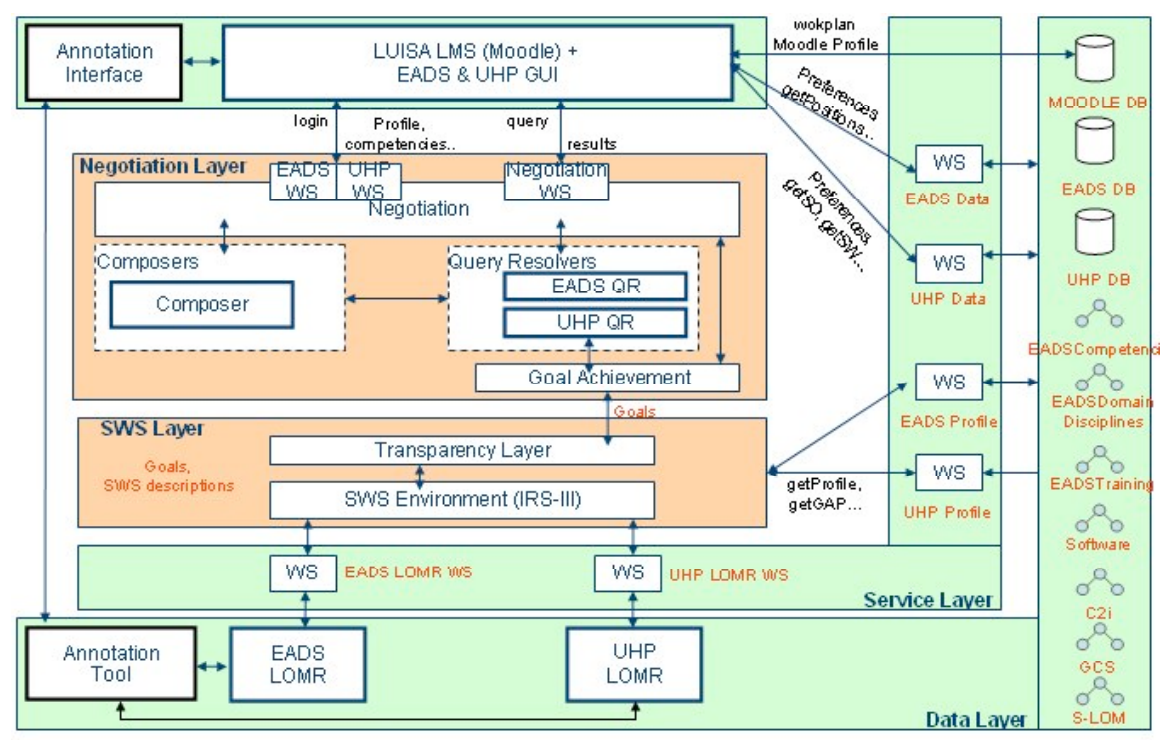

Fig. 1: Layered General Architecture proposed by LUISA.

\subsection{Shared Domain Models}

Domain models are provided as ontologies for describing a learning resource (based on the LOM standard) and for describing competencies. In LOM compliant metadata, the semantic interoperability is only performed through the meaning given to categories and through lists of values the semantic of which is often unclear, leading end-users to define application profiles. That is why, in LUISA, we propose a semantic version of LOM called s-lom.

This ontology is mostly based on the LOM schema to allow standard compliance as far as possible but it is designed to provide richer computational semantics. The Learning Object concept is modelled as a class, then the metadata attached to a LO are represented as the property values of a class instance. The value sets are extracted from the developed domain ontologies, which allows further reasoning on them. There is a growing demand of acquiring new competencies either in higher and professional education or in company training whereas learning resources are not all indexed in terms of competency acquisition. So in LUISA we propose a general competency ontology (GCO), derived from [19] each target application provides instances of competencies for enriching the description of resources and for performing competency based reasoning in order to fill competency gaps. The same ontology would also allow to shift from a competency based search to a subject matter based search when needed, as each competency definition is linked to one or several subject matter items. For example the competency instance "being able to use 
a textprocessor" is linked to the subject matter item "textprocessor", which in turn is exemplified by Word or OpenWriter in the computer literacy ontology.

\section{3 eLUISA, a Flexible Annotation Tool}

The flexibility of the knowledge framework would not be possible without flexibility in the annotation process. That is why the knowledge framework is completed with a flexible tool derived from the SHAME editor. eLUISA is configured for endusers through Annotation Profiles. An Annotation Profile (AP) is a configuration of the metadata editor defining what to edit and how to edit it. Indeed, more and more users participate through different roles in the edition of complex metadata coming from different metadata structures. Thus, APs allow to easily define in the editor which predefined values (from taxonomies or ontologies) should show up in which style (dropdown list, cursor to move, etc.) or how data types have to be checked. An administrator can compose an AP by creating and aggregating some modular bricks of annotation. For example, he can create a brick for the "language" metadata by associating a view (display a field entitled "Language") and a model (linking this field to the category 1.3 of LOM). Then, the administrator can decide to ask the annotators for competencies by adding into the AP a brick displaying a drop-down list dynamically filled with some instances of the competency ontology and associated to another structure than LOM.

\section{Customizing the Framework for a Target Application}

\subsection{Problem Addressed}

The general LUISA framework has to be customized for building working environments according to end-users needs. It has been applied to an academic environment and to an industrial environment. The academic prototype explores how semantic technologies could be used to discover the best suited Learning Objects for a given learner in a given domain. The chosen domain is computer and Internet litteracy as examplified in a French diploma called C2I. The main competencies are organized as follows (each competency is further subdivised into subcompetencies):

General and transversal competencies

A1: Be aware of the evolution of IT.

A2: Understand the ethical issues.

Specific and instrumental competencies

B1: Control his environment of work.

B2: Search for information.

B3: Save, secure and back-up his data in a local place or on a network.

B4: Build documents for printing.

B5: Build presentations of his work offline and online. 
B6: Communicate remotely.

B7: Produce a joint project.

The environment should allow the learner:

To express a query by exploring a set of competencies and additional criteria.

To obtain the more appropriate resources retrieved from one or several Learning Object Repositories (LOR) specially gathered and indexed for the prototype To be provided with tentatively packaged resources. The system can compose some Learning Objects (LO) to create a new one.

\subsection{The Customized Prototype Architecture}

The specific components, data structures and interfaces developed or adapted for the LUISA-UHP environment are labelled UHP on figure 1. They are summarized in Table1.

Table 1. Resources customized or designed for the LUISA-UHP environment

\begin{tabular}{|c|c|c|}
\hline Resource & Nature & Description \\
\hline $\mathrm{GCO} / \mathrm{C} 2 \mathrm{i}$ & Ontology & $\begin{array}{l}\text { Represents the competencies used in the C2I context. } \\
\text { These competencies are based on the concepts of the } \\
\text { General Competency Ontology. } \\
\text { Example: "k_email" is aKnowledgeElementDefinition } \\
\text { required by the "B6 competency: communicate } \\
\text { remotely" which is a CompetencyDefinition. }\end{array}$ \\
\hline $\begin{array}{l}\text { Computer Literacy } \\
\text { Ontology }\end{array}$ & Ontology & $\begin{array}{l}\text { Represents the items (hardware or software) involved } \\
\text { in the C2I competencies. } \\
\text { Example:"k_email" is a KnowledgeElementDefinition } \\
\text { about "EmailApplication" which is a } \\
\text { "PrivateCommunicationTool" in the Computer } \\
\text { Literacy Ontology. }\end{array}$ \\
\hline Discipline Ontology & Ontology & $\begin{array}{l}\text { Represents the fields of study in the university. } \\
\text { Example: "Medicine" is a specialization of "Health" } \\
\text { and is "linked to" "Biology". }\end{array}$ \\
\hline LOM/WSML & Ontology & $\begin{array}{l}\text { LOM/WSML is a semantic implementation of LOM. } \\
\text { It represents all the aspects (from technical } \\
\text { requirements to rights management and educational } \\
\text { characteristics) of a Learning Object. }\end{array}$ \\
\hline UHP Profile & Database & $\begin{array}{l}\text { MySQL database including all the other data about } \\
\text { UHP users. }\end{array}$ \\
\hline C2i LOs repository & LOMR & Including all LOs metadata about C2I. \\
\hline
\end{tabular}

The SWS infrastructure and the Annotation tool have to be integrated into a Learning Content Management System to allow users access the semantic functionalities supported by the core components of the architecture. For the first prototype an extension of the Open Source Moodle LMS has been developed. We also notice that some additional information about the user is needed for the UHP use case with respect to the information of the standard Moodle user profile, such information comprises: user competencies, user preferences about operating systems, 
available software, discipline, university preferences about cost and language. The additional information is stored in local databases.

\section{A Scenario Step by Step}

In this section we provide a scenario, focusing on the learner interaction with the prototype. The scenario is the following: a learner wants to find a suitable package of Learning Objects that suits his needs. The learners will use the prototype on their own, making use of the LUISA-based application in order to create a work plan to increase their competencies. For his first visit the learner has to describe his practical environment (Which operating system, which software suite, etc...) which will be stored in the UHP local database.

\section{Step 1 - Login and first set C2I competencies}

The learner logs in the LUISA UHP application and, once authenticated in Moodle, the prototype shows the learner his set of competencies according to his profile. The learner may select the competencies he wants to reach as illustrated in figure 2 and sends the query to the Negotiation Layer. The C2I competencies are presented as a dynamic expansible/ /collapsible tree showing the competencies he acquired and the missing ones. The tree is dynamic; when the user selects a competency new competencies (depending on the selected one) become available. The user can also select the duration he desires to practice.

\section{Step 2 - Rules for negotiation}

When the learner posts the request, the Negotiation Layer sends it to the SWS Layer by choosing the appropriate goal. The first invocation of the SWS layer tries always to retrieve LOs for the same competency and discipline that the learner provides. The Query Resolver component is in charge of analysing the response for all queries performed to the SWS Layer, trying to check the suitability of the selection. In the current prototype we have provided a specialization of the Query Resolver for the UHP case study that implements the following rules:

$\mathrm{R} 1$. If the competency chosen by the learner is a sub-competency:

R1.1 If there are no LOs that fulfill the exact match: The system selects the LOs about the same sub-competency but with a more general discipline. 


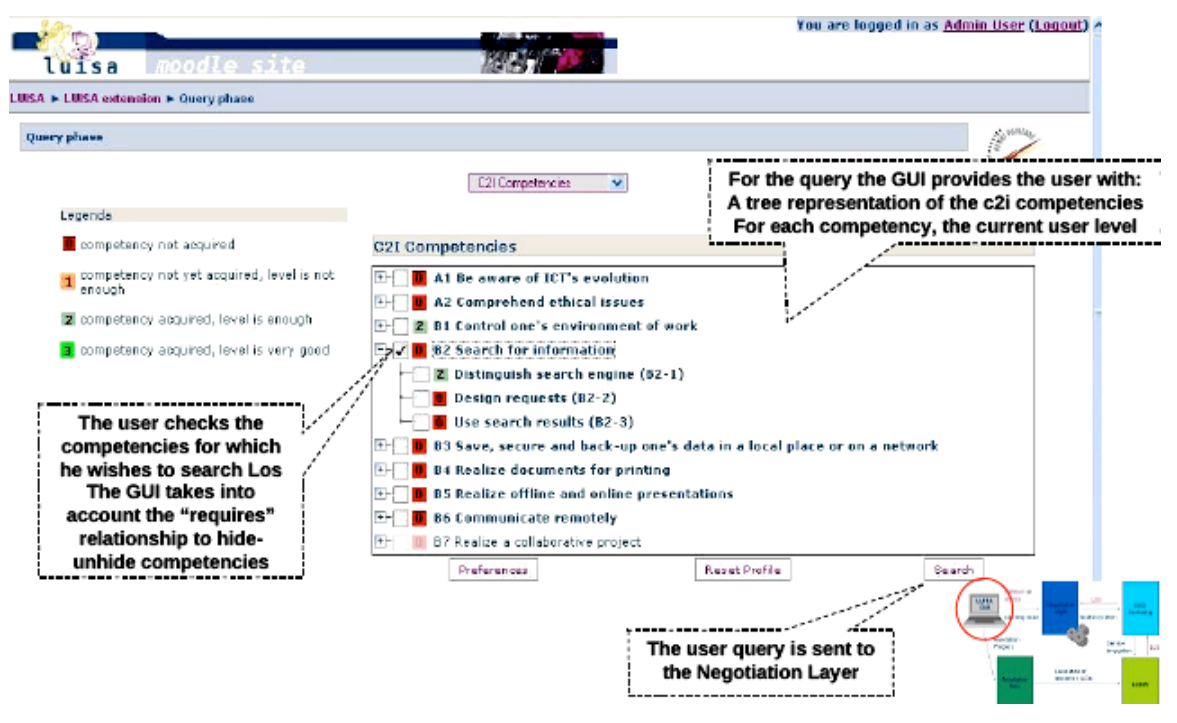

Fig. 2 : Target competencies displayed according to a learner profile

Application of organizational specific e-Learning rules.

R1.2 If there are no LOs with these features: The system selects the LOs about the general competency and the same discipline.

R1.3 If there are no LOs with these features: The system selects the LOs about the general competency and a more general discipline.

$\mathrm{R} 2$. If the student chooses a general competency:

If there are no LOs that fulfill the exact match: The system selects a LO about the same competency and a more general discipline + the LOs about the same discipline but whose target competency are sub-competencies included in the selected general competency.

The result of this phase is a set (possibly empty) of resources that fulfil the request as exemplified in figure 3. On the figure one can notice two kinds of ranking: the first one is related to the adequacy of the resource to the query, for instance a resource that does not cover all the subject have only 2 stars, the second one takes into account peers'rating, that is how useful the resource was for peer students, for instance a resource is possibly very useful for medecine students but not for students in history! Further developments about social rating are described in [5].

\section{Step 3 - Rules for composition}

The Composer component is in charge of creating learning packages by combination of a set of Learning Objects. The implementation of the composer for the current prototype assumes that the LOs are SCORM compliant and the resulted package is delivered as a new SCORM compliant LO. If the Query Resolver proceeds to a decomposition of a competency given by the end-user into a set of sub- 


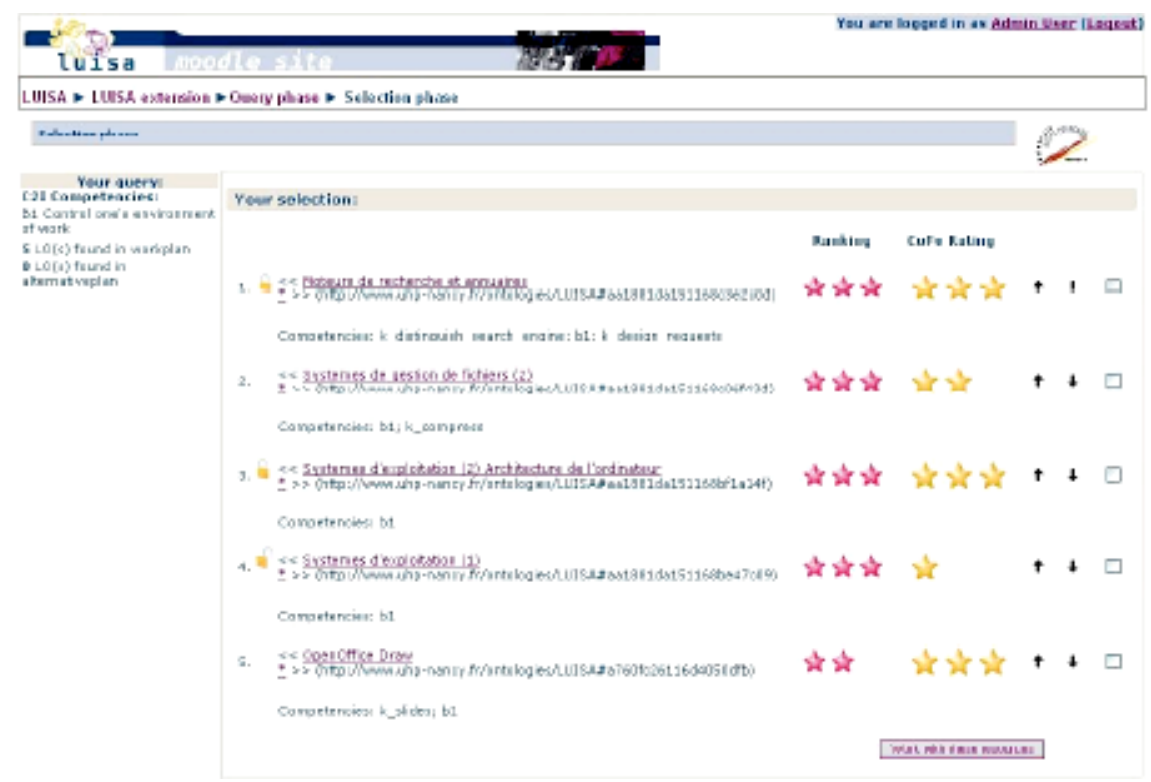

Fig. 3: LUISA-UHP selection

competencies, then the Composer gathers and packs the results for each subcompetency into a set that, as a whole, matches the supercompetency.

\section{Step 4 - Workplan}

Once the user selects the appropriate LOs, the work plan is saved in Moodle as the selection made by the user. Then the user has to download the selected resources. After completing the work, his profile will be updated.

\section{Users Feedback, Related Work and Future Trends}

The LUISA-UHP prototype has been proposed to academic staff, librarians and students. Teachers and trainers would like to use the system for selecting resources they would provide to their own students. They are interested in the packaging of resources and asked for more parameters (indeed the implemented criteria are very simple and merely provided as a proof of concept). They are also interested in more explanations on how the system has selected the resource (exact match, versus subcompetencies computations versus shift from competencies to topics). From a pedagogical point of view, the exploration and interactions with the tree of competencies is expected to improve the user's perception of the learning domain(meta-cognition). The librarians underline that the indexation process is not heavier that a LOM based one and the result is far better. Students were interested by a better harvest than with a google based search.

We have demonstrated that a semantic based environment allows to create an 
efficient competency based learning resources retrieval system. Many other on going projects aim at enhancing the description, the retrieval and reuse of appropriate learning objects by using semantic web technologies [11], [2], [3], [8]. Among them, LORNET [17] also proposes an ontology and a web service based architecture in its TELOS component. Another nation wide project in Korea [1] proposes the same kind of common knowledge framework but does not uses at all the flexibility provided by SWS and WS.

None of those systems is really similar to LUISA-UHP which

- Is a framework for developing specialized systems or brokers for other systems

- Enables exploring a set of competencies and the expression of queries in terms extracted from ontologies.

- Locates the best sources/providers for given queries (given learning needs and a learner profile).

- Suggests tentative compositions based on learning needs.

- Enables different query resolution/composition strategies, including educational

knowledge.

- Searches over several LORs using manual ontology alignment

- Takes into account peer rating

- Is extensible in terms of addition of new competencies or new topics, all existing rules and services remaining unchanged.

As building an ontology is time-consuming and as sharing ontologies will enhance interoperability between LORs, one of the next steps in our view is to cooperatively build, document and broadcast ontologies for the education world. Examples are to be found in the INTERGEO [6] project for geometry teaching, the LT4eL [10] project also about resource retrieval, but making an intensive use of language technologies, the Share.Tec project [18] for teacher training in Europe or the OMNIBUS [15] project including an educational theories ontology.

\section{Acknowledgements}

This paper benefits from the work done in the LUISA project co-funded by the UE (contract IST-FP6-027149). The author thanks all the partners for their contribution to the LUISA results reported in this paper.

\section{References}

1. Chang, B., Ham, D., Moon, D., Choi,, Y.-S, Cha, J. Using ontologies to search learning resources, in O. Gervasi and M. Gavrilova, (Eds.): ICCSA 2007, LNCS 4705, Part I, pp. 1146-1159, (2007)

2. Draganidis, F., Chamopoulou, P., Mentzas, G. An ontology based tool for competency management and learning paths. IKNOWS 06 Conference Proceedings, (2006)

3. Gasevic, D., Jovanovic, J., Devedzic, V. Ontology-Based Annotation of Learning Object Content, Interactive Learning Environments, 15 (1),1-26, (2007)

4. GLOBE, http://globe.edna.edu.au/globe/go.

5. Huynh-Kim-Bang, B., Dane, E. Social bookmarking tool based on structurable tags for communities of teachers, Young Researchers Track ITS'08, Montréal, (2008) 
6. I2G project web site : http://www.inter2geo.eu/

7. IMS consortium, http://www.imsglobal.org/digitalrepositories/index.html.

8. Kiu, C. C., Lee C. S. Ontology Mapping and Merging. through OntoDNA for Learning Object Reusability, Educational Technology \& Society, 9 (3), 27-42, (2006)

9. IEEE LOM specification: http://ltsc.ieee.org/wg12.

10. T4LeL web site : http://www.lt4el.eu/index.php?content=home.

11. Lefebvre, B., Gauthier, G., Tadie Guepfu, S., Duc, T., Achaba, H. Competence Ontology for Domain Knowledge Dissemination and Retrieval, Applied Artificial Intelligence 19 (910), 845-859, (2005)

12. LUISA Consortium: http://www .luisa-project.eu.

13. MERLOT, http://www.merlot.org.

14. OECD, Giving Knowledge for Free - The emergence of open educational resources, CERI publications, OECD pub, 146 p., www.oecd.org/publishing.

15. OMNIBUS ontology web site : http://edont.qee.jp/omnibus/doku.php

16. Open Courseware Consortium, http://www.ocwconsortium.org.

17. Paquette, G. An Ontology and a Software Framework for Competency Modeling and Management. Educational Technology \& Society, 10 (3), 1-21, (2007)

18. Share.Tec project web site $:$ http://www.share-tec.eu/

19. Sicilia M.A. Ontology based competency management: Infrastructures for knowledge intensive learning organization. In M.D. Lytras \& A. Naeve (eds) Intelligent learning infrastructure for knowledge intensive organizations: A semantic Web Perspective, p. 302324, Hershey, PA, Idea Group pub. (2005) 